\title{
Modeling Anisotropic Plasmon Excitations in Self-Assembled Fullerenes
}

\author{
Andrii Iurov $^{1}$, Godfrey Gumbs ${ }^{1,2}$, Bo Gao ${ }^{1}$ and Danhong Huang ${ }^{3}$ \\ ${ }^{1}$ Department of Physics and Astronomy, Hunter College of the City \\ University of New York, 695 Park Avenue, New York, NY 10065, USA \\ 2 Donostia International Physics Center (DIPC), \\ $P$ de Manuel Lardizabal, 4, 20018 San Sebastian, Basque Country, Spain \\ ${ }^{3}$ Air Force Research Laboratory, Space Vehicles Directorate, Kirtland Air Force Base, NM 87117, USA
}

(Dated: August 31, 2021)

\begin{abstract}
The plasmon excitations in Coulomb-coupled spherical two-dimensional electron gases (S2DEGs) reveal an interesting dependence on the displacement vector between the centers of the spheres with respect to the axis of quantization for the angular momentum quantum number $L$. Specifically, plasmon modes for a bundle of three S2DEGs have been obtained within the random-phase approximation (RPA). The inter-sphere Coulomb interaction matrix elements and their symmetry properties were also investigated in detail. The case of a bundle gives an adequate picture of the way in which the Coulomb interaction depends on the orbital angular momentum quantum number $L$ and its projection $M$. We concluded that the interaction between the S2DEGs aligned at an angle of $45^{\circ}$ with the axis of quantization is negligible compared to the interaction along and perpendicular to the quantization axis, which are themselves unequal to each other. Consequently, the plasmon excitation frequencies reveal an interesting orientational anisotropic coupling to an external electromagnetic field probing the charge density oscillations. Our result on the spatial correlation may be experimentally observable. In this connection, there have already been some experimental reports pointing to a similar effect in nanoparticles.
\end{abstract}

PACS numbers: 73.20.-r, 73.20.Mf, 78.20.Bh, 78.67.Bf

\section{INTRODUCTION}

The year 1995 was the culmination of several years of the search for fullerene materials. $\underline{\underline{1}-\underline{7}}$ It also marks the beginning of a whole new area of investigation into the optical and thermal properties of fullerene aggregates. From both an experimental and theoretical perspective, this branch of condensed matter physics is receiving international attention. Consequently, the theory for the properties of interacting fullerenes is a subject of considerable interest. $\underline{\underline{8}} \underline{\underline{10}}$

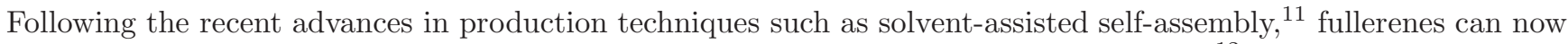
be produced in specific quantities even to form thin films of fullerene-like MoS nanoparticles $\frac{12}{2}$ and those achievements have stimulated renewed interest in these materials. Additionally, the ability to control optical fields has now made it feasible to ascertain the plasmon excitations in pre-arranged arrays of fullerenes $\stackrel{11,12}{12}$ The present paper has been stimulated by these exciting new developments, even though it employs a relatively simple model which is reasonable when the separation between the energy bands is large. $\underline{\underline{13}}$ Additionally, our results may serve as a guide for explaining experimental studies. $\stackrel{14.15}{ }$ Specifically, we are interested in calculating the plasma excitations of Coulomb coupled spherical two-dimensional electron gases (S2DEGs). $\underline{16}-19$ Here, we model three coupled spherical shells as shown in Fig.[1. In our discussion below, we refer to the S2DEG at the origin as "1"', and the one centered on the $x$ and $z$-axis as "2" and " 3 ", respectively. This notation then allows us to label the Coulomb matrix elements between spherical shells " $i$ " and " $j$ " with subscript " $i-j$ ", where $i, j=1,2,3$.

Electron energy loss spectroscopy (EELS) has been used to probe the plasmon excitations for concentric-shell fullerenes embedded in a film ${ }^{20}$ Furthermore, perfectly spherical shells were used in the theoretical modeling of the EELS data and the agreement was good. The model of Lucas, et al $\stackrel{23}{23}$ was shown to be qualitatively adequate for

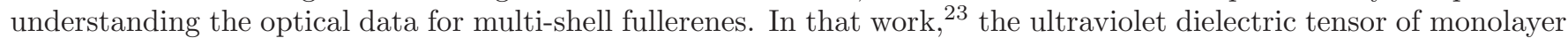
graphene is adapted to the spherical geometry of a fullerene by averaging over the three possible orientations of the c axis. Thus, a continuum model was used by Lucas, et al $\stackrel{23}{2}$ starting from the planar local di electric function of planar monolayer graphene.

Each S2DEG may be polarized by external electromagnetic fields. However, the S2DEG is only polarized for finite angular momentum quantum number $L \neq 0$. Although $L$ is a good quantum number for labeling the plasma excitations on an isolated shell, it is not the case for displaced, coupled S2DEGs. In contrast to the well-understood situation of determining the plasma excitations in fullerenes, $\underline{\underline{13}}$ we seek its properties when the rotational symmetry is broken. Here, for simplicity, $L=0$ corresponds to a non-circularly-polarized probe field, while $L=1$ is associated with a circularly-polarized probe field. Moreover, the higher angular momentum with $L>1$ may be achieved by a 
special light beam, e.g., a helical light beam. However, when two S2DEGs have their centers well separated so that there is no overlap of their charge distributions, the breaking of the spherical symmetry leads to significant differences with the multi-shell buckyball. Similar calculations of the polarization functions proved the existence of strongly localized image states near the surface of a buckyball21. Anisotropy of the plasmon interaction could be seen for two interacting shells 22

The topology of multiple shells forming an aggregate determines the properties of Coulomb interaction and consequently the details of the collective plasma excitations. For a single S2DEG, the Coulomb interaction depends on the angular momentum quantum number $L$ which in itself together with the radius of the shell serves to define the frequency of the plasmon mode. In contrast, when a pair of S2DEGs are coupled for a configuration such as the one exhibited in Fig.1 the polarization functions for all values of $L$ on each sphere may not be dissociated from one another. The inter-sphere Coulomb matrix element depends on both $L$ and its projection $M$ on the axis of quantization. Therefore, in principle, the plasmon mode equation is given in terms of a determinant of infinite order. But, for three interacting S2DEGs, the associated matrix may be broken down into diagonal sub-matrices corresponding to $L=1,2,3, \cdots$ and consisting of $3(2 L+1) \times 3(2 L+1)$ elements whose Coulomb interactions depend on $L$ and $(2 L+1)$ values of $M$ for each of the three shells. In contrast, the off-diagonal sub-matrices depend on Coulomb matrix elements which are functions of pairs of different angular momenta, $L$ and $L^{\prime}$, arising from each sphere. These off-diagonal Coulomb terms are generally smaller than their diagonal counterparts and so may be formally treated as perturbations. Consequently, in the lowest-order approximation, the $L=1$ mode is split by Coulomb interactions depending on $M=0, \pm 1$ from each sphere, leading to the occurrence of nine plasmon branches. However, some of these may be very close to each other depending on the strength of the Coulomb interaction. For example, when two S2DEGs are aligned along the $x$-direction, the number of well-separated plasmon branches is three and for the $z$-alignment we found only two non-equivalent plasmon branches.

In Sec.II] we will first formulate the method for calculating the plasmon equations on three coupled S2DEGs. This is based on the random-phase approximation (RPA) in evaluating the induced density fluctuations for a weak external perturbation. Section III is devoted to a discussion of our numerical results. Some concluding remarks are given in Sec.IV.

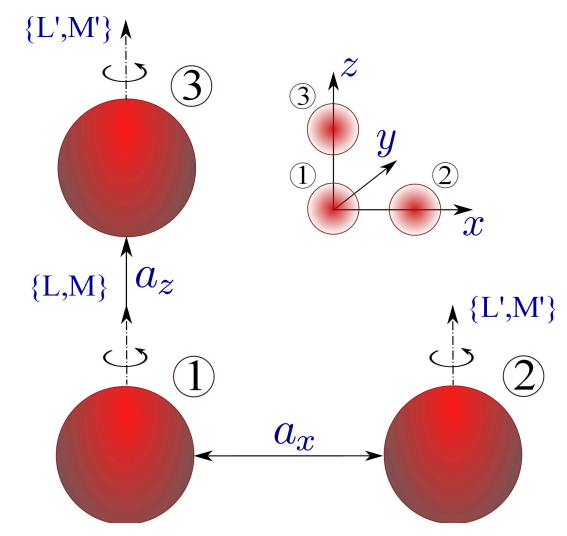

FIG. 1: (Color online) Schematic illustration of a triad of displaced S2DEGs. The axis of quantization is along the $z$ direction with angular momentum quantum number $L$ and component $M$. One shell has its center at the origin and the others are centered on the $x$ and $z$ axes.

\section{GENERAL FORMULATION OF THE PROBLEM}

Let us consider a bundle consisting of three spherical shells with their centers located at the origin, on the $x$ axis and the $z$ axis. That is, the center of one of the spheres is at $\mathbf{r}_{1}=0$ with radius $R_{1}$ whereas the others are centered at $\mathbf{r}_{2}=(a, 0,0)$, with radius $R_{2}$ and $\mathbf{r}_{3}=(0,0, a)$, with radius $R_{3}$. We assume that the inequalities $a>R_{1}+R_{2}$ and $a>R_{1}+R_{3}$ are satisfied. In the absence of tunneling between the shells, the wave function for an electron on the $j$-th shell $(j=1,2,3)$ is given by

$$
<\mathbf{r} \mid j \nu>=\Psi_{j l m}\left(\vec{r}-(3-j)(j-1) a \hat{e}_{x}-\frac{1}{2}(j-1)(j-2) a \hat{e}_{y}\right), \quad \Psi_{j l m}(\vec{r})=f_{j}(r) \frac{1}{\sqrt{R_{j}^{2}}} Y_{l m}(\Omega)
$$


in terms of spherical harmonics $Y_{l m}(\Omega)$ with $\nu=\{l, m\}$ and $f_{j}^{2}(r)=\delta\left(r-R_{j}\right)$. The energy spectrum has the form of $\epsilon_{j, \nu}=\hbar^{2} l(l+1) /\left(2 m^{*} R_{j}^{2}\right)$. The induced potential $\Phi$ satisfies Poisson's equation $\nabla^{2} \Phi(\mathbf{r}, \omega)=\left(4 \pi e / \varepsilon_{s}\right) \delta n(\mathbf{r}, \omega)$, where $\varepsilon_{s} \equiv 4 \pi \epsilon_{0} \varepsilon_{b}$ and $\varepsilon_{b}$ is the uniform background dielectric constant. We use linear response theory to calculate the induced particle density $\delta n(\mathbf{r}, \omega)$. After a straightforward calculation, we obtain

$$
\sum_{j^{\prime}=1}^{3} \sum_{L^{\prime}=0}^{\infty} \sum_{M^{\prime}=-L^{\prime}}^{L^{\prime}}\left[\delta_{j j^{\prime}} \delta_{L L^{\prime}} \delta_{M M^{\prime}}+\frac{2 e^{2}}{\varepsilon_{s}} \Pi_{j^{\prime}, L^{\prime}}(\omega) V_{j^{\prime} L^{\prime} M^{\prime}, j L M}\left(R_{j}, R_{j^{\prime}}, a\right)\right] U_{j^{\prime}, L^{\prime} M^{\prime}}=0
$$

where

$$
\begin{aligned}
\Pi_{L}(\omega) & =\sum_{l, l^{\prime}} \frac{f_{0}\left(\epsilon_{l}\right)-f_{0}\left(\epsilon_{l^{\prime}}\right)}{\hbar \omega+\epsilon_{l^{\prime}}-\epsilon_{l}}(2 l+1)\left(2 l^{\prime}+1\right)\left(\begin{array}{ccc}
l & l^{\prime} & L \\
0 & 0 & 0
\end{array}\right)^{2}, \\
V_{j^{\prime} L^{\prime} M^{\prime}, j L M}\left(R_{j}, R_{j^{\prime}} ; a\right) & =8 \int \frac{d^{3} \mathbf{q}}{q^{2}} j_{L}\left(q R_{j}\right) j_{L^{\prime}}\left(q R_{j^{\prime}}\right) Y_{L M}^{*}(\hat{\mathbf{q}}) Y_{L^{\prime} M^{\prime}}(\hat{\mathbf{q}}) \\
& \times e^{i\left[(3-j)(j-1) q_{x} a+\frac{1}{2}(j-1)(j-2) q_{y} a\right]} e^{-i\left[\left(3-j^{\prime}\right)\left(j^{\prime}-1\right) q_{x} a+\frac{1}{2}\left(j^{\prime}-1\right)\left(j^{\prime}-2\right) q_{y} a\right]}
\end{aligned}
$$

with $j_{L}(x)$ being a spherical Bessel function and $\Pi_{j, L}(\omega)$ being the density response function of the $j$-th nano shell. Also, we have introduced the quantity

$$
U_{j, L M}=\frac{1}{L_{x} L_{y} L_{z}} \sum_{q_{x}, q_{y}, q_{z}} e^{i\left[(3-j)(j-1) q_{x} a+\frac{1}{2}(j-1)(j-2) q_{y} a\right]} \frac{\delta n\left(q_{x}, q_{y}, q_{z}, \omega\right)}{q_{x}^{2}+q_{y}^{2}+q_{z}^{2}} j_{L}\left(q R_{j}\right) Y_{L M}^{*}(\hat{\mathbf{q}})
$$

Here, $L_{x}, L_{y}$ and $L_{z}$ are normalization lengths. Thus, non-trivial solutions for the charge density oscillations correspond to the zeros of the determinant of the coefficient matrix for $U_{j, L M}$ in Eq. (2).

\section{NUMERICAL RESULTS}

In Fig.2, we compare the strengths of the electrostatic interaction between pairs of S2DEGs when $L=0, M=0$ and $L=1$ with $M=0, \pm 1$, where all the non-zero Coulomb matrix elements for an interacting triad of spheres displayed schematically in Fig.1 are presented. The matrix elements $V_{L M, L^{\prime} M^{\prime}}$ with $L=0$ and $L^{\prime}=0$, which do not contribute to plasmon excitation, are also provided for comparison. These matrix elements greatly exceed all the others because of rotational symmetry.

Our calculations show that the Coulomb interaction is significant only at distances that are comparable to the sum of the radii of the two interacting spheres. In the notation of Fig. 1 we have shown that the $(2-3)$ interaction is negligible compared to the $(1-2)$ and $(1-3)$ interactions. The reason for this difference is not due to the larger separation between $(2-3)$ which is $\sqrt{2}$ times that between $(1-2)$ or $(1-3)$ but it is a consequence of the angular dependence inherent in the matrix elements. As a matter of fact, unlike the $(1-2)$ and $(1-3)$ matrix elements, we are unable to obtain semi-analytic expressions for the $(2-3)$ Coulomb matrix elements since even the angular part of the related Coulomb integral cannot be expressed in analytical form. Using numerical methods, however, we have estimated these matrix elements to be on the order of $10^{-2}$ times smaller than all the rest. This results in the existence of one strong nearly-degenerate plasmon mode, which is practically independent of separation $a$ between the centers of the spheres. Additionally, due to the reduced symmetry, there are more non-zero matrix elements for $(2-3)$ coupling compared to the $(1-2)$ and $(1-3)$ cases, which however does not improve the impact of $(2-3)$ interaction because of their relatively small magnitude.

Setting $L, L^{\prime}=1$ and $M, M^{\prime}=0, \pm 1$ in the set of linear equations (2) for $j=1,2,3$, leads to a $9 \times 9$ matrix of the coefficient matrix of $\left\{U_{j, L M}\right\}$. The dephasing constant $\delta$ is chosen to be $0.05 \mathrm{eV} / \hbar$. We present density plots in the following figures for the inverse of this dielectric function to demonstrate the plasmon modes of interacting S2DEGs.

Now, let us consider three identical S2DEGs with equal radius $R_{1}=R_{2}=R_{3}$ at the corners of a right-angle triangle as shown in Fig.1. Numerical results for the plasmon and particle-hole frequencies are presented in Fig. 3 , where we only chose $L, L^{\prime}=1$ and $M, M^{\prime}=0, \pm 1$. Generally, the number of plasmon branches is determined by the 


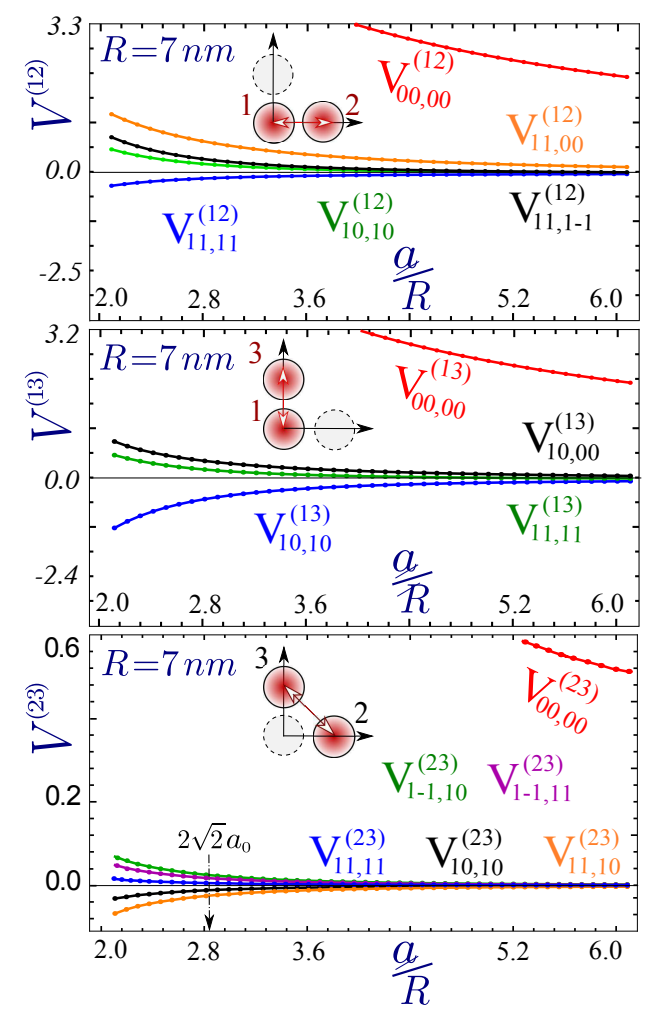

FIG. 2: (Color online) Coulomb matrix elements, given in units of $2 e^{2} /\left(\pi \epsilon_{s} R\right)=0.59 \mathrm{eV}$, for the various interactions between spheres (a) "1" and "2", (b) "1" and "3" and (c) "2" and "3". The radii of the S2DEGs are equal with $R=7 \mathrm{~nm}$. The inter-sphere distance $a$ is given in units of $R$.

dimensionality of the truncated matrix, with $2 L+1$ solutions associated with each sphere. We separate the case of two spheres with $z$-alignment $(1-3)$, for which the interaction sub-matrix is diagonal with two unequal non-zero elements, and the whole matrix allows exact diagonalization. Such interaction results in two symmetrically split pairs of plasmon branches, presented in Fig. 3 (a1) and (b1). All other plots in Fig.3 show the plasmons for three interacting S2DEGs. We expect nine separate plasmon branches, corresponding to the solutions of $\left.\Re e\left[\epsilon_{\Delta}\right](R, \omega)\right]=0$. However, three of them, corresponding to the $(2-3)$ interaction, are nearly-degenerate, due to its low relative strength of the Coulomb coupling. Apart from that, one or a few solutions, located far away from the central branch, are very weak and could not be shown for the chosen range of frequencies. Plots (a3) and (b3) in Fig. 3 present both plasmons and particle-hole modes, as the peaks of $1 / \Re e\left[\epsilon_{\Delta}(\omega, R)\right]$ and $\Im m\left[\Pi^{0}(\omega, R)\right]$ correspondingly. We calculated those quantities over a wider range of frequencies for different values of radius $R$ and separation $a$. We conclude that the plasmons and single-particle excitation regions are well-separated so that the plasmons are not Landau damped for all cases of an interacting bundle. But, the electron-hole mode region and plasmons get closer with increasing radius of the shell.

Our results for plasmon excitations for three interacting S2DEGs with unequal radii are presented in Fig. (4) We chose the radius of the sphere at the origin (sphere 1) to be larger than that of either sphere 2 or 3 whose radius is still chosen to be equal to one another. i.e., $R_{1}>R_{2}=R_{3}$. We note that in this case the ratio of the Coulomb interaction between spheres $(2-3)$ to that between either $(1-2)$ or $(1-3)$ is even smaller, compared to the same ratio of three S2DEGs with the same radius $R_{1}=R_{1}=R_{3}$ since the distance between shells $(1-2)$ and $(1-3)$ is increased.

When the separation $a$ between spheres $(1-2)$ or $(1-3)$ becomes large compared to $R_{1}$, the plasmon frequencies when plotted as a function of $a$ form two horizontal branches, with each one arising from the plasmons on each isolated sphere with radii $R_{1}$ and $R_{2}$. The higher frequency branch is due to the two spheres with smaller radius $R_{2}=R_{3}$. As a consequence, this plasmon branch exhibits a stronger resonance peak with a higher degree of degeneracy. The three solutions, corresponding to the $(2-3)$ interaction, stay nearly degenerate for all values of the separation $a$. This behavior is similar to the previous case when $R_{1}=R_{2}=R_{3}$. The resulting plasmon branches split apart, creating three asymmetric pairs of solutions. This is a general feature. 

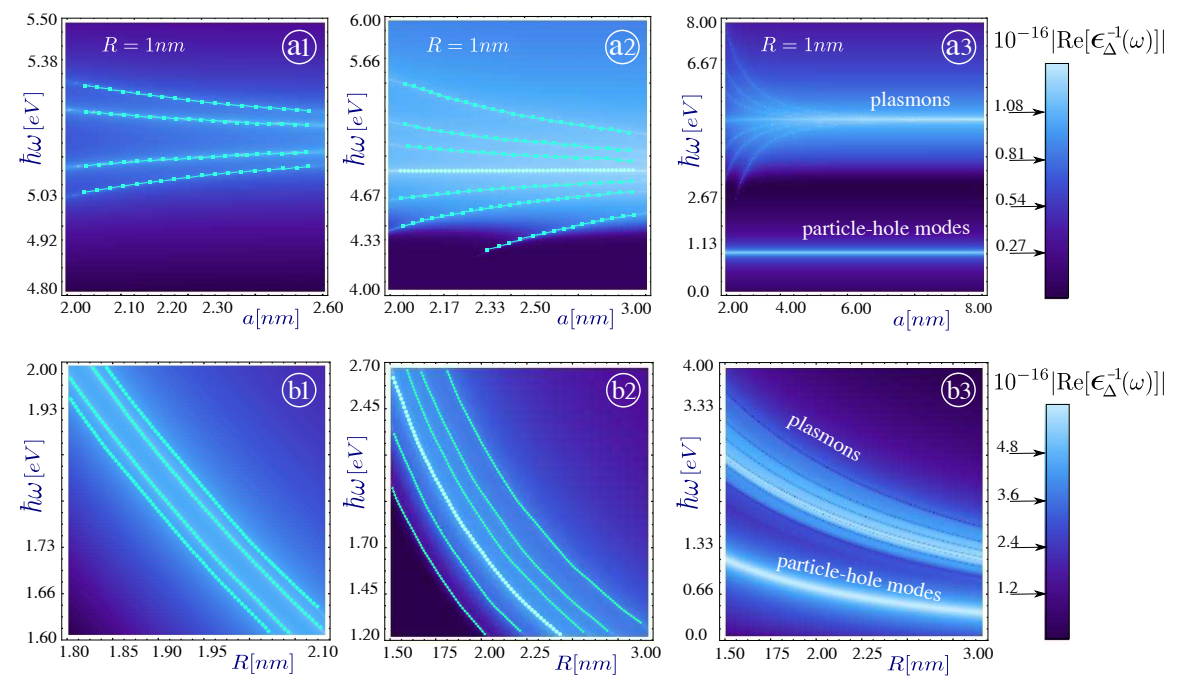

FIG. 3: (Color online) Density plot of the inverse of the dielectric function $\epsilon_{\Delta}(R, \omega)$, defined to be the determinant of the coefficient matrix in Eq. (2), demonstrating the plasmon modes of interacting spheres. The upper row [plots (a1)-(a3)] shows how the frequencies of the excitations depend on the separation $a$ between the centers of the shells with equal radius $R=1 \mathrm{~nm}$. These three panels highlight the plasmon spectrum for different ranges of the variable $a$. The lower panel presents the plasmon frequency dependence on the radius of each S2DEG. Plots (a1) and (b1) show the four plasmon branches for the two spheres $(1-3)$, on the $z$-axis, for which the matrix could be diagonalized exactly. Plots (a2) and (b2) show the plasmon branches for three interacting S2DEGs, including the horizontal nearly-degenerate subset of branches, resulting from (2-3) interaction. All the above mentioned plots also include numerical solutions for the plasmons, corresponding to the zeros of the dielectric function. Panels (a3) and (b3) present all the plasmon branches of an interacting S2DEGs over a wider range of separation and frequencies. The number of occupied energy levels used in these calculations is $N_{F}=8$ for all six panels.
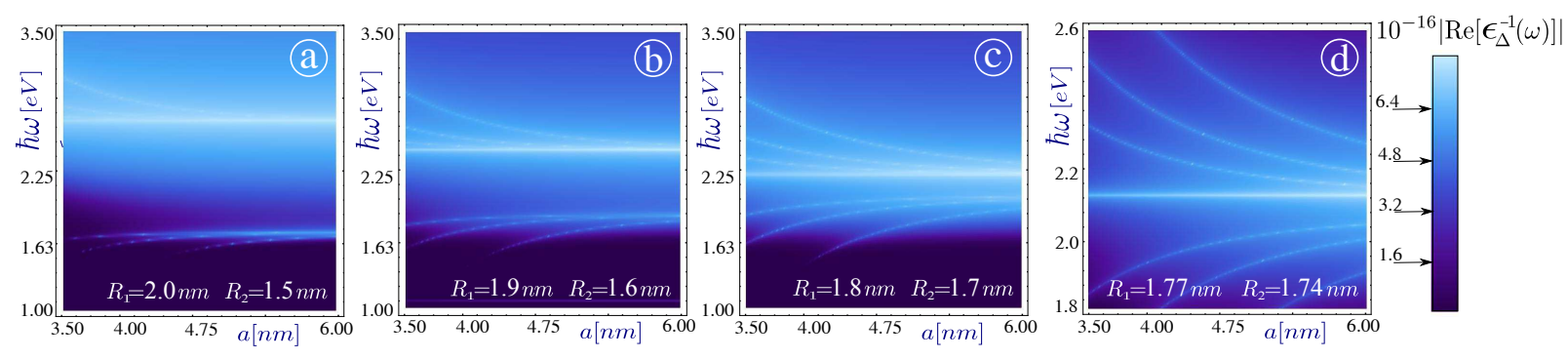

FIG. 4: (Color online) Density plot of the inverse dielectric function vs. the separation between their centers. Each plot corresponds to a separate case of two unequal radii of the sphere located at the origin (1) and two others with $R_{1} \neq R_{2}=R_{3}$. The plasmon branches correspond to the maxima of $1 / \Re e\left[\epsilon_{\Delta}\right]$. We chose the density such that the number of occupied levels is $N_{F}=9$ for all spheres. Also, $L=1$ as the only value of the angular momentum, contributing to plasmon excitations. All plasmon excitations merge to form a single branch for $a \gg R$.

Figure 5 compares the plasmon excitations for the $(1-2)$ and $(1-3)$ pairs of S2DEGs. From the results presented here, we conclude that we have clearly demonstrated the anisotropy of the plasmon mode excitations for a particulate. The sum-rule presented by Apell, Echenique, and Ritchie 24 for surface plasmon frequencies does not apply here. Although the surfaces in our model exhibit spatially localized collective modes, the plasmons for an isolated S2DEG may be labeled by a single quantum number $L$ but, when the S2DEGs are coupled, all angular momentum quantum numbers and their projections onto the axis of quantization determine the plasmon frequencies. However, we emphasize that these plasma frequencies for the $(1-2)$ and $(1-3)$ configurations are functionals of the plasmon frequency $\Omega_{L}$ of a single S2DEG, and consequently must be related to each other. The frequency $\Omega_{L}$ is determined by the solutions of the equation $\Re e\left[1-e^{2} /\left(\epsilon_{s}(2 L+1) R\right) \Pi_{L}\left(\Omega_{L}\right)\right]=0$. Due to the diagonal nature of the Coulomb interaction submatrices for the $(1-3)$ case of $z$-alignment, the equations for the plasmon frequencies may be reduced to $\operatorname{Re}\left[1-e^{2} /\left(\epsilon_{s}(2 L+1) R\right) \Pi_{L}\left(\omega_{z}\right) \pm v_{1,3}(a) \Pi_{L}\left(\Omega_{z}\right)\right]=0$, leading to the two pairs of solutions which obviously depend on $\Omega_{L}$. For the case of $(1-2) x$-alignment, the Coulomb potential matrix elements contain a larger number of non-zero teems and, more importantly, this submatrix is not diagonal. Consequently, the plasmon equation may not be presented in such a simple functional form. Complexity of the resulting plasma equation does not prevent 
numerical solution but clearly highlights the difference in the plasmon frequencies for $x$ and $z$-alignments.

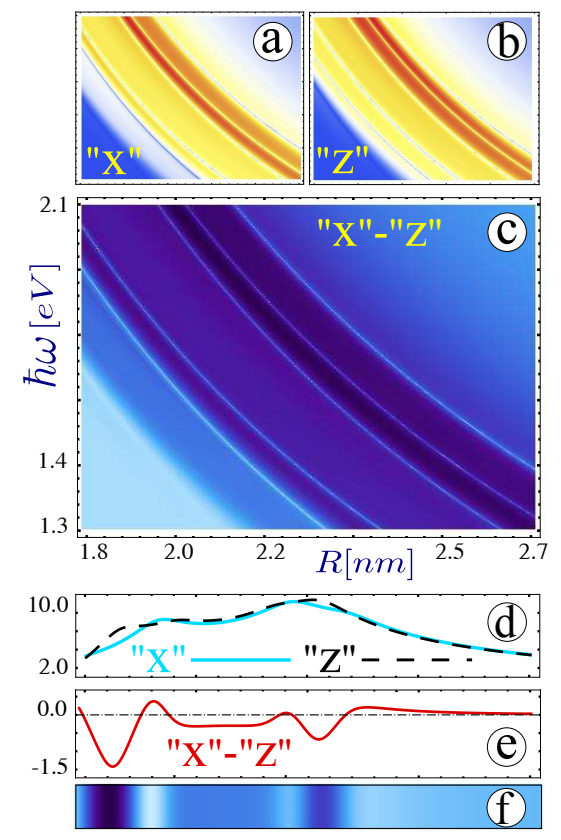

FIG. 5: (Color online) Comparison of plasmon excitations for the $(1-2)$ and $(1-3)$ pairs of S2DEGs. Panels (a) and (b) are density plots for the inverse dielectric function for the $(1-2)$ and $(1-3)$ interacting S2DEGs, respectively. Plot (c) shows the difference between the absolute values obtained in (a) and (b) over the same range of radius $\left(R_{1}=R_{2}=R\right)$ and plasmon frequencies $\left(\omega_{\min }=1.3 \mathrm{eV} / \hbar\right.$ and $\left.\omega_{\max }=2.1 \mathrm{eV} / \hbar\right)$. Panel $(\mathrm{d})$ shows the $R$-dependence of the inverse dielectric function for chosen frequency $\omega=1.50 \mathrm{eV} / \hbar$, which is outside the frequency range of panel (c). Panel (e) demonstrate the difference between these results in $(d)$. The results presented in the form of $\ln (1+\Pi)$ in order to minimize the difference between the lowest and the highest values. Panel (f) represents the density plot, for the preceding plot (e), which also could be considered as the colorbar for panel (c).

\section{CONCLUDING REMARKS}

In this paper, we presented a theory for calculating the orientational dependence of coupled plasmon excitations on a pair of interacting S2DEGs. Though the model is simple, we demonstrated that it is a powerful tool for exhibiting the novel orientational dependence of plasmon excitations. The energy band structure is a suitable approximation for fullerenes when the energy bands are far apart. However, realistic energy band structure may be incorporated into the polarization function through a form factor involving the wave functions of the electrons as well as the Coulomb matrix elements.

We note that spectral correlations have been observed experimentally for metallic nanoparticles $\underline{25}$ In that work, the plasmons for pairs were studied using polarization-selective total internal reflection. Their measurements show that the frequencies for the coupled plasmon modes depend on whether the incident light wave vector perpendicular and parallel to the dimer axis. Related work on dimer plasmons has been conducted by Nordlander, et al. (Ref.[26]) with the conclusion that the hybridized plasmon energy arising from individual metallic nanoparticles is determined by the orientation of the inter-particle axis with respect to the axis of polarization of the two constituents modeled as incompressible spherical liquids. Although our model differs from that in Ref. 26, 27] the conclusions about the existence of anisotropy in the plasmon excitation energies in these systems are in agreement. Similar effects are also expected to be observed in the case of nano-eggs: non-concentric multishells of nanoparticles. The hybridization of the plasmons has been proven to be an adequate and precise method to describe the plasmonic structure $\underline{\underline{28,29}}$. The field enhancement, corresponding to the resonant excitation of plasmons, was reported to be much larger in the case of concentric nanoparticles, which support indirectly the concept of plasmon spatial correlation.

Since the plasmon dependence on the relative orientation and the separation between the interacting spheres was the principal property under investigation, we considered an aggregate of three which includes coupling along, perpendicular and making an angle with the axis of quantization for angular momentum. We clearly established 
the orientational dependence of the coupled plasmon excitation frequencies arising from the sensitive nature of the Coulomb matrix elements. Our formalism allows for any number and location of the S2DEGs, thereby leading to possible studies of aggregates of different size and shape.

Once the angular momentum quantum number $L$ is specified, the number of plasmon branches is determined by all the different non-zero potential matrix elements. The number of those elements is a consequence of symmetry properties. Specifically, when a pair of spheres $((1-3))$ are aligned along the axis of quantization, there are only two such non-zero matrix elements, whereas three of them exist for $(1-2)$ interaction between the spheres, aligned along the $x$-axis. There are five non-zero Coulomb matrix elements between spheres whose centers lie on a line making an angle of forty-five degrees with the axis of quantization, but much smaller in magnitude. Additionally, the effect of the Coulomb interaction on the plasmons of a smaller sphere, occurring at a higher frequency, is just a small perturbation for $(2-3)$ coupling. Our conclusion is that the unique characteristic has high potential for electronics, sensing applications and photoelectric conversion.

\section{Acknowledgments}

This research was supported by contract \# FA 9453-07-C-0207 of AFRL. DH would like to thank the support from the Air Force Office of Scientific Research (AFOSR).

1 E. Osawa, Kagaku (Kyoto) 25, 854 (1970) [in Japanese].

2 J. F. Anacleto and M. A. Quilliam, Anal. Chem. 65, 2236 (1993).

${ }^{3}$ H. W. Kroto, J. R. Heath, S. C. O'Brien, R. F. Curl and R. E. Smalley, Nat. 318, 162 (1985).

4 S. Iijima, J. Crystal Growth 50, 675 (1980).

5 P. R. Buseck, S. J. Tsipursky and R. Hettich, Sci. 257, 215 (1992).

6 J. Cami, J. Bernard-Salas, E. Peeters and S. E. Malek, Sci. 329, 180 (2010).

7 C. A. Poland, R. Duffin, I. Kinloch, A. Maynard, W. A. H. Wallace, A. Seaton, V. Stone, S. Brown, W. MacNee and K. Donaldson, Nat. Nanotechn. 3, 423 (2008).

8 T. Inaoka, Surf. Sci. 273, 191 (1992).

9 J. Tempere, I. F. Silvera and J. T. Devreese, Phys. Rev. B 65, 195418 (2002).

10 C. Yannouleas, E. N. Bogachek and U. Landman, Phys. Rev. B 53, 10225 (1996).

11 Lang Wei, Jiannian Yao, and Hongbing Fu, ACS Nano, 7 (9), pp 75737582 (2013).

12 Manish Chhowalla and Gehan A. J. Amaratunga, Nature 407, 164 (2000).

13 D. Östling, P. Apell and A. Rosen, Europhys. Lett. 21, 539 (1993).

14 G. Gensterblum, J. J. Pireaux, R. Gaudano, J. P. Vigneron, A. A. Lucas and W. Krätschmer, Phys. Rev. Lett. 67, 2171 (1991).

15 E. Schmen, J. Fink and W. Krätschmer, Europhys. Lett. 17, 51 (1992).

16 V. K. Voora, L. S. Cederbaum and K. D. Jordan, J. Phys. Chem. Lett. 4, 6 (2013).

17 A. K. Belyaev, A. S. Tiukanov, A. I. Toropkin, V. K. Ivanov, R. G. Polozkov and A. V. Solovýov, Physica Scripta 80, 048121 (2009).

18 P. Longe, Solid State Commun. 97, 857 (1996).

19 M. T. Michalewicz and M. P. Das, Solid State Commun. 84, 1121 (1992).

${ }^{20}$ L. Henrard,* F. Malengreau, P. Rudolf, K. Hevesi, R. Caudano, and Ph. Lambin, Th. Cabioch, Phys. Rev. B 59, 5832 (1999).

21 Godfrey Gumbs, Antonios Balassis, Andrii Iurov, and Paula Fekete, The Scientific World Journal, vol. 2014, 726303 (2014).

22 Godfrey Gumbs, Andrii Iurov, Antonios Balassis, Danhong Huang; arXiv:1309.7328v3 [cond-mat.mes-hall].

23 A. A. Lucas, L. Henrard, and Ph. Lambin, Phys. Rev. B 49, 2888 (1994).

24 S. P. Apell, P. M. Echenique, and R. H. Ritchie, Ultramicroscopy 65, 53 (1996).

25 Yang, Shu-Chun and Kobori, Hiromu and He, Chieh-Lun and Lin, Meng-Hsien and Chen, Hung-Ying and Li, Cuncheng and Kanehara, Masayuki and Teranishi, Toshiharu and Gwo, Shangjr, Nano Letters, 10, 2 (2010).

26 Nordlander, P. and Oubre, C. and Prodan, E. and Li, K. and Stockman, M. I., Nano Letters, 4, 5 (2004).

27 Prodan, E. and Radloff, C. and Halas, N. J. and Nordlander, P., Science, 302, 5644 (2003).

28 Y.Wu and P.Nordlander, The Journal of Chemical Physics, 125, 125, 124708 (2006).

29 Bardhan, Rizia and Mukherjee, Shaunak and Mirin, Nikolay A. and Levit, Stephen D. and Nordlander, Peter and Halas, Naomi J., The Journal of Physical Chemistry C, 114, 16 (2010). 